\section{Indicadores de salud oral en niños y adolescentes con parálisis cerebral de centros especializados de Lima- Perú, periodo 2012- 2013}

\author{
Oral health indicators in children and \\ adolescents with cerebral palsy from \\ specialized centers of Lima- Peru, 2012- \\ 2013 period
}

\section{Resumen}

Objetivo. Evaluar indicadores de salud oral en niños y adolescentes con parálisis cerebral en Lima-Perú. Métodos. Estudio observacional, descriptivo y transversal de una muestra de 171 pacientes de dos centros de referencia nacional especializados, periodo 20122013, que cumplieron los criterios de inclusión. Para los índices de higiene oral (IHO) de Greene \& Vermillion, índice de dientes cariados, perdidos y obturados (CPOD) y análisis de maloclusión dentaria (MD) se evaluaron 126 niños (6,0-11,9 años) y 45 adolescentes (12,0-19,0 años). Resultados. El IHO fue de 2,55 (malo) en el 91,8\%, IHO regular en el $8,2 \%$ y ninguno mostró IHO bueno, observándose el mismo comportamiento en ambos grupos etarios. La prevalencia de caries fue $100 \%$ y CPOD de $4,55 \pm 1,56$; los niños registraron CPOD de 4,52 $\pm 1,537$ y los adolescentes CPOD de 4,64 $\pm 1,626$. El $74,3 \%$ mostró MD, los niños evidenciaron MD en el $69,8 \%$ y los adolescentes mostraron $86,7 \%$. En los niños, se observó relación molar (RM) Clase I derecha en el 42,1\% e izquierda en el 44,4\%; los adolescentes evidenciaron RM Clase III derecha en el 42,2\% e izquierda en el $37,8 \%$. Para los niños, el overbite (OB) no fue registrable en el 33,3\% y normal en el 29,4\%; en adolescentes, se registró OB normal en el 40\%. Se observó con mayor frecuencia línea media dentaria desviada hacia el lado izquierdo. Conclusiones. En la mayoría de niños y adolescentes con parálisis cerebral de centros especializados se encontró alto índice de caries CPOD, mala higiene oral y presencia de maloclusiones.

Palabras clave: Índice CPO; Índice de higiene oral; Maloclusión; Parálisis cerebral (fuente: DeCS BIREME).

\begin{abstract}
Objective. To evaluate oral health indicators in children and adolescents with cerebral palsy in Lima-Peru. Methods. An observational, descriptive and cross-sectional study of a sample of 171 patients from two specialized national referral centers for the 20122013 period who met the inclusion criteria. For the Greene \& Vermillion oral hygiene index $(\mathrm{OHI})$, decayed, missing and filled teeth (DMFT) and the dental malocclusion (DM) analysis, 126 children (6.0-11.9 years-old) and 45 adolescents (12.0-19.0 yearsold) were evaluated. Results. The OHI was 2.55 (poor hygiene) in the $91.8 \%$, regular $\mathrm{OHI}$ in the $8.2 \%$ and no one showed a good $\mathrm{IHO}$, observing the same behavior in both age groups. The prevalence of caries was $100 \%$ and the DMFT was $4.55 \pm 1.56$; the
\end{abstract}

ISSN-L 1560-9111; eISSN: 1609-8617

Artículo Original

María Angélica Alvarez-Páucar 1,a, María del Rocío Lu-Chang Say ${ }^{2, b}$, Roxana Revoredo-Morote ${ }^{3, c}$

${ }^{1}$ Universidad Nacional Mayor de San Marcos, Facultad de Odontología, Departamento Académico de Estomatología Pediátrica, Lima, Perú.

2 Universidad Peruana Cayetano Heredia, Facultad de

Estomatología, Departamento Académico de Medicina y Cirugía Buco Maxilofacial, Lima, Perú.

${ }^{3}$ Universidad Nacional Mayor de San Marcos, Facultad de Odontología, GI YATIRIS- UNMSM, Lima, Perú.

${ }^{a}$ Especialista en Odontopediatría. Doctor en Ciencias de la Salud.

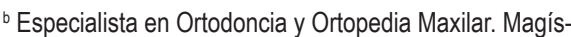
ter en Estomatología.

${ }^{c}$ Especialista en Ortodoncia y Ortopedia Maxilar. Master en Gerencia Pública.

\section{Correspondencia:}

María Angélica Alvarez-Páucar: malvarezp1@unmsm. edu.pe

Av. Germán Amezaga N ${ }^{\circ} 375$ - Ciudad Universitaria 3er. Nivel Cercado de Lima, Lima Metropolitana, Perú. Lima 01

ORCID: 0000-0003-4337-0127

\section{Coautores:}

María del Rocío Lu-Chang Say: maria.lu.chang@upch.pe ORCID: 0000-0003-4076-1202

Roxana Revoredo-Morote: roxrevoredo@hotmail.com ORCID: 0000-0002-4733-3545

\section{Editor:}

Guido Artemio Marañón Vásquez

Universidade Federal do Rio de Janeiro, Brasil

Conflicto de intereses: María Angélica Alvarez-Páucar es editora adjunta de la revista Odontología Sanmarquina

Fuente de financiamiento: CONCYTEC

\section{Recibido: $26 / 11 / 20$}

Aceptado: $27 / 02 / 21$

Publicado: 01/07/21 
children registered a DMFT of $4.52 \pm 1.537$ and the adolescents $4.64 \pm 1.626$. The DM was present in $74.3 \%$; the children showed a $69,8 \%$ of DM and the adolescents showed $86.7 \%$. In children, a right class I molar relationship (MR) was observed in $42.1 \%$ and a left MR in 44.4\%; the adolescents showed right Class III in $42.2 \%$ and left in $37.8 \%$. In children, the overbite $(\mathrm{OB})$ was not registered in the $33.3 \%$ of the cases and it was normal in the $29.4 \%$; the adolescents registered a normal OB in $40 \%$. It was more frequently observed a deviation of the dental midline to the left. Conclusions. In most children and adolescents with cerebral palsy from specialized centers, a high index of DMFT caries, poor oral hygiene and the presence of malocclusions were found.

Keywords: Cerebral palsy; DMF index; Malocclusion; Oral hygiene index (source: MeSH NLM).

\section{Introducción}

La parálisis cerebral (PC), es uno de los trastornos neurológicos más frecuentes de la discapacidad motora en la edad pediátrica ${ }^{1,2}$, considerado como un problema de salud pública a nivel mundial ${ }^{3,4}$. Según la Red de Vigilancia de PC en Europa (SCPE), lo define, como un grupo de trastornos permanentes, pero no cambiantes del movimiento y/o postura y de la función motora, que se deben a una interferencia no progresiva, lesión o anormalidad del cerebro en desarrollo o inmaduro; excluye los trastornos progresivos de la función motora, definidos como pérdida de habilidades adquiridas previamente a los 5 ańos de vida, excluyendo a los niños con hipotonía como única característica clínica y con defectos aislados del tubo neural espinal ${ }^{5}$. Su etiología es compleja, se basa según momento de aparición: prenatal, perinatal y posnatal, relacionados con hipoxia cerebral infantil en el periodo perinatal; nuevos estudios observaron que los factores prenatales (curso del embarazo) son responsables del $75 \%$ de los casos, mientras que los factores de riesgo del período infantil y neonatal representaron entre el 10 a $18 \%$; además la corta duración del embarazo y bajo peso al nacer son considerados factores determinantes ${ }^{6}$.

Las tasas de prevalencia pueden variar según ámbito geográfico, la revisión mundial del 2013 halló, que la prevalencia global de PC fue de 2,11/1000 nacidos vivos ${ }^{7}$; el estudio de la Universidad de Washington reveló una prevalencia mundial baja, alrededor del $1 \%$, notándose un mayor registro en niños que en niñas 1,5: $1 / 1000$ nacidos vivos, con una elevada incidencia en poblaciones africano-americanas ${ }^{8}$. Su incidencia oscila entre el 2\% de los nacidos vivos en países desarrollados y 2,5 a 5 casos por 1000 nacidos vivos en los países en desarrollo ${ }^{4}$. En Europa, la prevalencia es decreciente, 1,77/ 1000 niños $^{9,10}$. La clasificación de PC, se puede agrupar en 04: espástica, discinética/atetoide, atáxica y mixta ${ }^{8}$.

El estado oral de los pacientes con PC, evidencian algún tipo de alteración en las funciones de respiración, masticación, deglución y fonación. Los reflejos primitivos del lactante con PC como: succión, deglución, reflejo perioral, reflejo faríngeo y mordisqueo son importantes, además pueden causar deglución atípica y anomalías dento-maxilares ${ }^{11}$. Los estudios en la población general reportan presencia o no de maloclusión dentaria (MD) 12,13 , formas erradas de mordida que no proveen estética, ni función masticatoria correcta y desequilibran el rostro $^{13}$. Para establecer el diagnóstico, se analizan los modelos de estudio y la boca en oclusión ${ }^{14}$, sin embargo, en trabajos previos desarrollados en pacientes con PC, sólo se utilizaron el análisis clínico de maloclusiones 11,15 , tomando de referencia los planos del espacio y/o la Relación Molar de Angle, posición sagital de los primeros molares permanentes considerados puntos estables de la arquitectura craneofacial 12,16,17. Aunque ninguna patología oral específica está asociada con PC, las mismas enfermedades que afectan a la población en general, puede presentarse en estos pacientes vulnerables con una mayor gravedad debido a su discapacidad motora, evidenciándose mayor riesgo a desarrollar enfermedades prevalentes como caries dental, enfermedad gingival, entre otras ${ }^{15,18,19}$; varios autores revelan una higiene oral deficiente observada en personas con PC, debido a la dificultad de cepillarse los dientes ${ }^{15,18,20}$, por ende, la instalación temprana de caries dental. La revisión de literatura nacional, refiere escasos estudios sobre este tipo de discapacidad y sus patologías orales. La presente investigación, tiene como propósito, determinar algunos indicadores de salud oral que presentan los niños y adolescentes con PC procedentes de centros especializados, lo que permitirá conocer la situación de salud y desarrollar a futuro, programas de atención de salud oral.

\section{Métodos}

La investigación fue observacional, descriptivo y transversal. Se calculó un tamaño muestral de 136 pacientes con PC para una población de 212 (calculada en base a Tabla de Daños Principales que causan discapacidad en el ciclo de vida hasta los 19 años de edad, INR 2011) ${ }^{21}$. La muestra estuvo conformada por 171 nińos y adolescentes con PC, de los centros de referencia nacional, Instituto Nacional de Rehabilitación - "Adriana Rebaza Flores" (INR) y Hogar Clínica San Juan de Dios (HCSJD) durante el periodo 2012- marzo 2013. La presente investigación forma parte de un estudio principal, utilizándose su Big Data. Se cumplió con las normas éticas de la Declaración de Helsinki- Acta de Evaluación Ética Aprobada Nº292, Facultad de Medicina (Universidad Nacional Mayor de San Marcos. Los criterios de inclusión fueron: pacientes con PC como primer diagnóstico, 
sin otra discapacidad, sin alteración genética, malformaciones ni tumoraciones orales, no respiradores bucales, sin tratamiento ortodóncico y presencia de primeros molares permanentes, pacientes con asentimiento cuyos padres firmaron el consentimiento informado.

El trabajo se realizó en tres etapas, primero se llevó a cabo estrategias de aprestamiento, abordaje y triaje en la atención de salud de los pacientes, ejecutándose los índices de higiene oral. Luego, se evaluó el índice de caries CPOD y finalmente el análisis de la maloclusión. Este periodo sirvió para completar la muestra necesaria debido a la deserción (mayoría de edad o abandono del estudio). Para la evaluación de la higiene, se utilizó el índice de higiene oral de Greene \& Vermillion ${ }^{22}$, para la identificación de caries, se tomó el índice $\mathrm{CPOD}^{23}$ y para la evaluación clínica de maloclusiones, se llevó a cabo la clasificación topográfica ${ }^{12}$, que distingue tres tipos de maloclusiones según el plano del espacio (sagital, vertical y transversal). La investigadora principal (Maestría en Odontoestomatología de Salud Pública y Especialista en Odontopediatría), fue quien realizó las evaluaciones clínicas de los pacientes y requirió calibración en el área de ortodoncia para la evaluación de las maloclusiones aplicado en pacientes con discapacidad. Se calibró con un gold standard del área de ortodoncia (Coordinador de la Especialidad Estomatología de Pacientes Especiales y Especialista de Ortodoncia, RLCh-S), prueba inter-kappa: 0,82. El análisis de las maloclusiones consistió en el análisis vertical, sobremordida u overbite; el análisis transversal, línea media dentaria, mordida cruzada y mordida de Brodie, y por último, el análisis sagital, traspase horizontal $\mathrm{u}$ overjet $(\mathrm{OJ})$, relación canina $(\mathrm{RC}) \mathrm{y}$ relación molar $(\mathrm{RM})^{16}$.

Se tomó el examen visual/táctil, en un ambiente iluminado, empleando el sillón dental y luz artificial en el HCSJD, mientras que, en el INR, se utilizó una camilla y muebles del consultorio médico. Se emplearon dos espejos faciales y un frontoluz de dos intensidades (Energizer $\left.{ }^{\circledR}\right)$. Se utilizaron cajas estériles que contenían: seis espejos bucales $\mathrm{N}^{\circ} 5$ (Maillefer ${ }^{\circledR}$ ), seis sondas CPITN calibradas (Hu-Friedy $($ ) $)$, tres reglas milimetradas de metal, tres condensadores de metal No 40 con sus topes de caucho, tres pinzas para algodón, un porta-algodonero de metal, tres porta desechos, tres bandejas de plástico. Materiales fungibles, gasas estériles, algodón, hisopos, dos reveladores de placa líquida (Plac ${ }^{\bullet}$ Control (), dos enjuagues bucales de CPC 0,05\% + clorhexidina $0,05 \%$ mantenimiento / active control sin alcohol (Perio•Aid @), campos, vasos descartables, baja lenguas, sablón, uniforme pediátrico y equipo de protección personal.

Para la recolección de la data y análisis estadístico se uilizó el programa estadístico SPSS v20, mediante el análisis descriptivo y análisis bi-variado prueba Chi cuadrado con un $\mathrm{p}<0,05$.

\section{Resultados}

La muestra estuvo constituida de 171 pacientes con PC, cuyas edades comprendían entre los 6 a 19 años, provenientes de 02 centros especializados de Lima Metropolitana. Se presenta la descripción de los sujetos de estudio de ambas instituciones (Tabla 1), mostrando presencia mayoritaria del sexo masculino, asimismo, el grupo etario predominante fue el más joven (6-11,9 años) y sobre los tipos de PC, se observa principalmente al grupo espástico.

Respecto a la higiene oral, los 171 pacientes con PC, cuyas edades comprendían entre los 6 a 19 años, registraron el índice de higiene oral simplificado de Greene y Vermillion (IHO) que fue de 2,495 $\pm 0,36$, además se observa que en ambos grupos etarios la media de IHO fue similar de 2,492 $\pm 0,35$ para el grupo de 6-11,9 años y de 2,503 $\pm 0,39$ para el grupo de 12-19,0 años (Tabla 2). De acuerdo, a la categorización del IHO según grupo etario se registró mal o deficiente estado de higiene oral en el $91,8 \%$ de los casos, seguido del estado de higiene regular con el 8,2\% y ningún caso evidenció una higiene oral buena; asimismo ambos grupos etarios mostraron similar comportamiento registrándose para el grupo de 6,0- 11,9 años, el IHO malo en el 92,9\% y

Tabla 1. Descripción de los participantes con parálisis cerebral

\begin{tabular}{|c|c|c|c|c|c|c|}
\hline \multirow{3}{*}{ Características } & \multicolumn{4}{|c|}{ Institución } & \multirow{2}{*}{\multicolumn{2}{|c|}{ Total }} \\
\hline & \multicolumn{2}{|c|}{ HCSJD } & \multicolumn{2}{|c|}{ INR } & & \\
\hline & $\mathrm{n}$ & $\%$ & $\mathrm{n}$ & $\%$ & $\mathrm{n}$ & $\%$ \\
\hline \multicolumn{7}{|l|}{ Sexo } \\
\hline Masculino & 57 & 53,8 & 39 & 60,0 & 96 & 56,1 \\
\hline Femenino & 49 & 46,2 & 26 & 40,0 & 75 & 43,9 \\
\hline \multicolumn{7}{|l|}{ Grupo etario } \\
\hline 6- 11,9 años & 77 & 72,6 & 49 & 75,4 & 126 & 73,7 \\
\hline $12,0-19,0$ años & 29 & 27,4 & 16 & 24,6 & 45 & 26,3 \\
\hline \multicolumn{7}{|l|}{ Tipo de PC } \\
\hline Espástica & 86 & 81,1 & 46 & 70,8 & 132 & 77,2 \\
\hline Discinética & 8 & 7,5 & 7 & 10,8 & 15 & 8,8 \\
\hline Atáxica & 9 & 8,5 & 8 & 12,3 & 17 & 9,9 \\
\hline Mixta & 3 & 2,8 & 4 & 6,2 & 7 & 4,1 \\
\hline Total & 106 & 62,0 & 65 & 38,0 & 171 & 100 \\
\hline
\end{tabular}

HCSJD: Hogar Clínica San Juan de Dios. INR: Instituto Nacional de Rehabilitación 
el IHO regular en el 7,1\%, para el grupo de 12,0-19,0 años, se observó el IHO malo en el 88,9\% y el IHO regular en el 11,1\%. $(\mathrm{p}<0,05)$ (Tabla 3).

Respecto al estado de caries, se observó un índice CPOD de 4,55 correspondiéndole un valor alto de caries dental, teniendo un valor mínimo de 2 y máximo de 9 . El grupo etario de 6,0-11,9 años registró un CPOD de $4,52 \pm 1,537$, similar al valor obtenido por el grupo de mayor edad de 12,0-19,0 años con un CPOD de 4,64 $\pm 1,626$ ambas con estimación alta de caries. (Tabla 4). La prevalencia de caries en nuestra muestra de estudio fue del $100 \%$.

Respecto a la presencia de maloclusión dentaria (MD), se agrupó con MD (Clase II, Clase III y NR) en el $74,3 \%$ y sin MD (Clase I) en el 25,7\%; el grupo de niños (6,0-11,9 años) registró MD (Clase II, Clase III y NR) en el 69,8\% y sin MD (Clase I) en el 30,2\% y el grupo de adolescentes (12,0-19,0 años) mostró MD (Clase II, Clase III y NR) en el 86,7\% y sin MD (Clase I) en el 13,3\% (p: 0,027). En la relación molar (RM), se observó mayormente RM Clase I, relación molar derecha (RMD) en el 37,43\% y relación molar izquierda (RMI) en el 38,01\%; RM Clase II para la RMD el $32,75 \%$ y RMI el 29,82\%; RM Clase III para la RMD el $25,73 \%$ y RMI el $23,98 \%$. Observando que, el grupo de 6,0 -11,9 ańos obtuvo predominantemente RM Clase I para la RMD el $42,1 \%$ y RMI el $44,4 \%$; el grupo de 12,0-19,0 años mostró principalmente RM Clase III para la RMD el 42,2\% y RMI el 38\%; hallándose diferencia significativa para la RMD (p: 0,023) y RMI (p: 0,013) (Tabla 5).

Tabla 2. Análisis descriptivo del índice de higiene oral

\begin{tabular}{|c|c|c|c|c|c|c|}
\hline \multirow{2}{*}{ Grupo etario } & \multicolumn{6}{|c|}{ IHO } \\
\hline & Media & $\mathbf{n}$ & $\%$ & Des. Típ. & Mínimo & Máximo \\
\hline $6,0-11,9$ años & 2,492 & 126 & 73,7 & 0,35 & 1,5 & 3,0 \\
\hline $12,0-19,0$ años & 2,503 & 45 & 26,3 & 0,39 & 1,5 & 3,0 \\
\hline Total & 2,495 & 171 & 100,0 & 0,36 & 1,5 & 3,0 \\
\hline
\end{tabular}

IHO: Índice de Higiene Oral

Tabla 3. Valoración del IHO de niños y adolescentes con parálisis cerebral

\begin{tabular}{|c|c|c|c|c|c|c|}
\hline \multirow{3}{*}{ Grupo etario } & \multicolumn{6}{|c|}{ IHO } \\
\hline & \multicolumn{2}{|c|}{ Bueno } & \multicolumn{2}{|c|}{ Regular } & \multicolumn{2}{|c|}{ Malo } \\
\hline & $\mathbf{n}$ & $\%$ & $\mathbf{n}$ & $\%$ & $\mathbf{n}$ & $\%$ \\
\hline $6,0-11,9$ años & 0 & 0 & 9 & 7,1 & 117 & 92,9 \\
\hline $12,0-19,0$ años & 0 & 0 & 5 & 11,1 & 40 & 88,9 \\
\hline Total & 0 & 0 & 14 & 8,2 & 157 & 91,8 \\
\hline
\end{tabular}

IHO: Índice de higiene oral

Tabla 4. Índice CPOD de niños y adolescentes con parálisis cerebral

\begin{tabular}{lcccccc}
\hline \multirow{2}{*}{ Grupo etario } & & \multicolumn{3}{c}{ CPOD } \\
\cline { 2 - 6 } & Media & $\mathbf{n}$ & \% & Desv.Típ & Mínimo & Máximo \\
\hline $6,0-11,9$ años & $4,524,64$ & 126 & 73,7 & 1,537 & 2 \\
$12,0-19,0$ años & 45 & 26,3 & 1,626 & 2 \\
\hline Total & 4,55 & 171 & 100,00 & 1,557 & 2 \\
\hline
\end{tabular}

CPOD: Índice de caries, perdidos y obturados por diente

Tabla 5. Relación molar dentaria de niños y adolescentes con parálisis cerebral

\begin{tabular}{|c|c|c|c|c|c|c|c|c|}
\hline \multirow{3}{*}{ Grupo etario } & \multicolumn{8}{|c|}{ Relación molar dentaria } \\
\hline & \multicolumn{4}{|c|}{ Relación molar derecha } & \multicolumn{4}{|c|}{ Relación molar izquierda } \\
\hline & $\mathrm{CI}$ & CII & CIII & NR & CI & CII & CIII & NR \\
\hline $6-11,9$ a. & $42,1 \%$ & $34,1 \%$ & $19,8 \%$ & $4,0 \%$ & $4,4 \%$ & $29,4 \%$ & $7,1 \%$ & $7,1 \%$ \\
\hline $12-19,0$ a. & $24,4 \%$ & $28,9 \%$ & $42,2 \%$ & $4,4 \%$ & $20,0 \%$ & $31,1 \%$ & $37,8 \%$ & $11,1 \%$ \\
\hline Total & $37,42 \%$ & $32,7 \%$ & $25,7 \%$ & $4,18 \%$ & $38,0 \%$ & $29,8 \%$ & $23,9 \%$ & $8,3 \%$ \\
\hline
\end{tabular}

Cl: Clase I, CII: Clase II, C III: Clase III, NR: No registrable 
En el overbite (OB), el grupo etario de 6,0-11,9 años de edad, mayoritariamente no fue registrable en el 33,3\%, seguido del $\mathrm{OB}$ normal. Por otro lado, el grupo etario de 12,0-19,0 años, observó principalmente $\mathrm{OB}$ normal en el $40,0 \%$, seguido del OB negativo (mordida abierta) en el $4,4 \%$. Hubo diferencia significativa entre los grupos (p: 0,001) (Figura 1). Aunque, el overjet, según sexo, edad, tipo de PC y centro especializado de procedencia no evidenció diferencia significativa entre los grupos.

Respecto a la línea media dental (LMD), se registraron tres categorías: línea media dental coincidente (LMD
C), línea media dental no coincidente (LMD NC) y los casos no registrables (NR). Se notó que el grupo con LMD NC sumó $60,8 \%$, de los cuales, la mayoría registró LMD desviada hacia la izquierda con el $41,5 \%$ y el $19,3 \%$ fue desviada hacia la derecha; mientras que el grupo de la LMD C, registró el 29,2\% de los casos. (Figura 2). Según grupo etario de 6,0 - 11,9 ańos de edad, observó en su mayoría LMD NC, con el 57,1\%; el grupo etario de 12,0 - 19,0 años de edad, siguió la misma tendencia, con el $71,1 \%$. Se observó diferencia significativa entre los grupos $(\mathrm{p}=0,028)$.

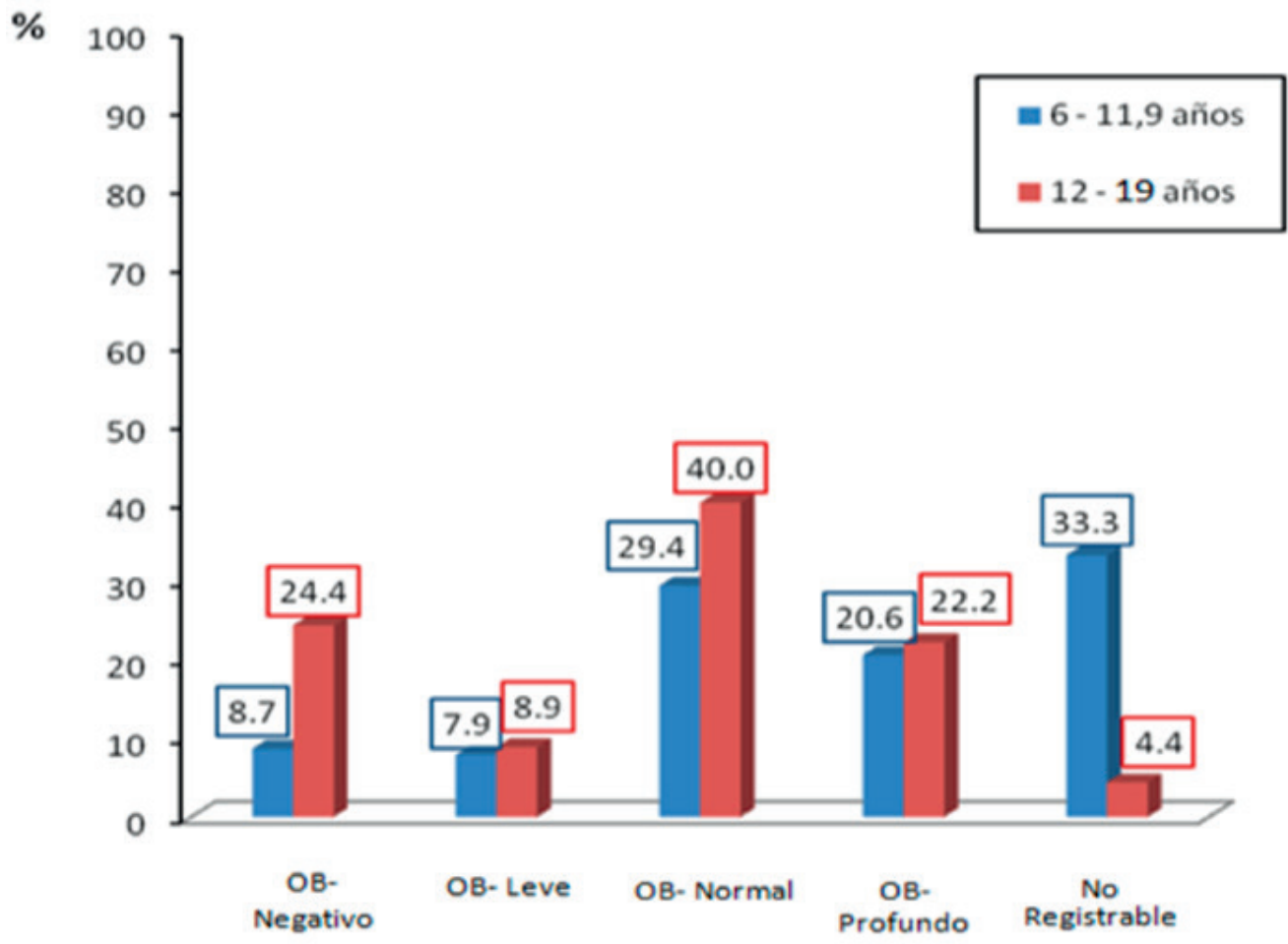

Figura 1. Overbite según grupo etario

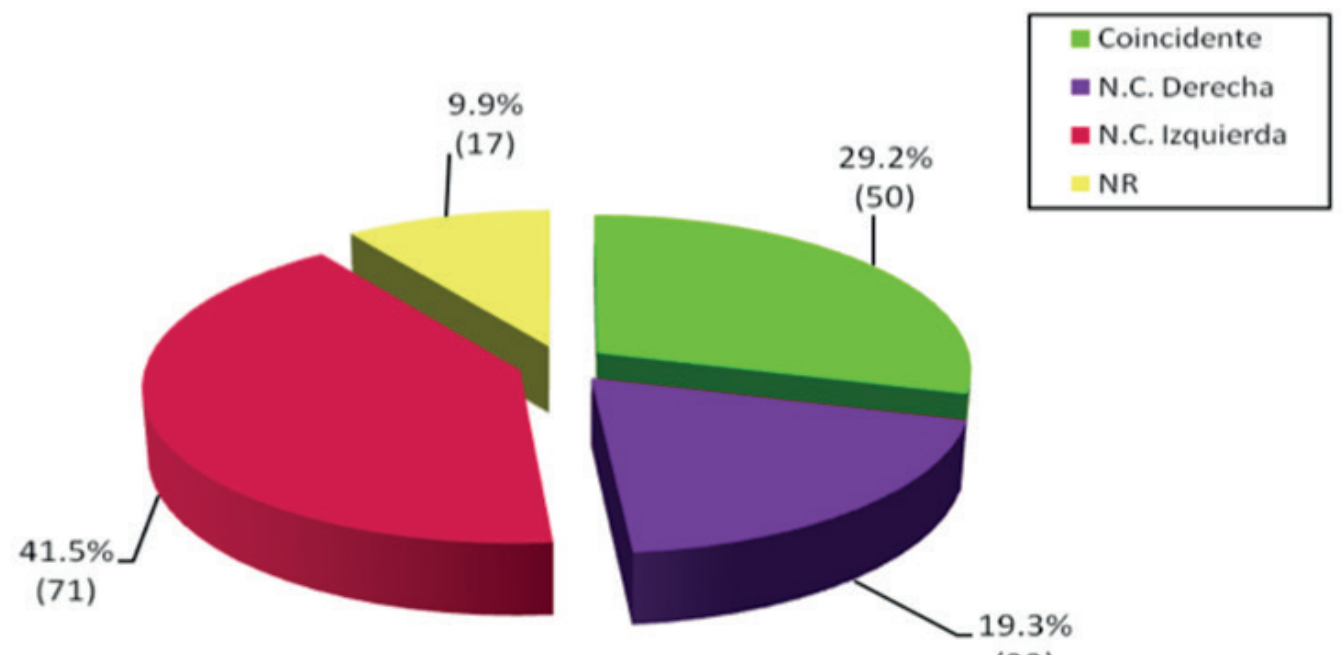

(33)

Figura 2. Frecuencia y porcentaje sobre la relación de las líneas medias 


\section{Discusión}

Según el último estudio del INR, los pacientes con PC en el Perú (2012) ${ }^{24}$, representaron el $12 \%$ del total de pacientes con discapacidad (muestra representativa de 400 casos) para los ańos $2006 \mathrm{al}$ 2008. La presente investigación tuvo una muestra representativa de $171 \mathrm{ca}-$ sos durante el periodo 2012- marzo 2013, es importante resaltar, que este tipo de pacientes requiere mucho apoyo emocional del núcleo familiar y acompañamiento durante sus citas de atención, su cooperación, su tolerancia al stress físico y emocional pueden estar alterados y/o disminuidos, reflejándose en el estrés de la cita dental ${ }^{15}$, ${ }^{25}$, por lo que, dificulta su abordaje para el diagnóstico, tanto médico como odontológico.

El presente estudio observó mayor tendencia a desarrollarse PC en el sexo masculino (M) que en el femenino (F) con el 56,1\%, similar resultado lo evidenciaron Barrionuevo \& Solís ${ }^{11}$, Guerreiro \& De Lima ${ }^{26}$, SabbaghHaddad mostrando una relación de 1,57/1: M/F ${ }^{27}$ y comparándolo con el estudio peruano de Soto et al. ${ }^{28}$, se observó el mismo comportamiento con el $60,6 \%$ en el grupo M. Respecto al grupo etario, los participantes de menor edad (grupo de 6,0-11,9 años) mostraron el $73,7 \%$, asimismo Guerreiro \& De Lima ${ }^{26}$, reportaron la misma tendencia en su estudio, el grupo de 1-5 años obtuvo el $51,2 \%$ y $48,8 \%$ el grupo de 6-12 ańos, probablemente sea la respuesta a su atención de salud integral temprana y oportuna. Esta investigación, reportó que el tipo de PC espástica fue de 77,2\%, seguido de la discinética, atáxica y mixta, similar disposición lo observó la Universidad de Washington ${ }^{8}$, a diferencia del estudio peruano de Soto et al. ${ }^{28}$, que evidenció diferente orden, la PC espástica fue de 75,9\%, luego mixta, atáxica y discinética/ atetósica.

En una primera etapa, se aplicó el IHO, indicador oral que surgió como una estrategia de abordaje conductual y aprestamiento para llevar a cabo sus evaluaciones clínicas. El presente estudio, reveló que el IHO fue malo en el $91,81 \%$ de los casos, sin embargo, Sinha et al. ${ }^{29}$ observó mayoritariamente un IHO regular en el $42 \%$ de la muestra, seguido del IHO bueno con el $30 \%$ y malo con el $28 \%$ de los casos, se presume que los centros contactados tuvieron intervenciones de promoción de la salud oral en algún momento. Cardoso et al. ${ }^{30}$ mostró una prevalencia del $94,73 \%$ de sangrado gingival para el grupo etario de 7-18 años evidenciando alteración del estado gingival por diversas razones, entre ellas: mala higiene oral debido a su condición, falta de supervisión en el cepillado, consumo de medicación y otros.

Respecto al índice de caries, la presente investigación evidenció registro alto del índice CPOD de 4,55 $\pm 1,557$, además la prevalencia de caries fue del $100 \%$. Cardoso et al..$^{30}$, observó también, una alta prevalencia de caries con el 71,3\%, sin embargo, obtuvo un CPOD de 1,71 $\pm 2,42$ correspondiéndole un valor bajo de caries. Por otro lado, Abanto et al. ${ }^{31}$ reportaron una prevalencia de caries del $55 \%$ e índice CPOD de 0,43 , reflejando valores bajos respecto a los anteriores estudios citados, probablemente se deba, a que el estudio fue realizado en pacientes atendidos en el Centro de Atención del Paciente Especial (CAPE-USP), donde fueron beneficiados de una atención primaria oportuna.

Respecto a la RM de Angle, el presente estudio mostró una mayor tendencia en la categoría con MD (Clase II y clase III) sobre la categoría sin MD (Clase I) como se evidenció en un anterior estudio de Álvarez ${ }^{32}$, pero sí se observa, por separado, que la Clase I obtuvo una leve ventaja sobre las otras relaciones molares. El grupo de niños registró una RM Clase I con el 42,1\% (RMD) y $44,4 \%$ (RMI), mientras que el grupo de adolescentes obtuvo principalmente Clase III con el 42,2\% (RMD) y $37,8 \%$ (RMI). Los resultados del grupo etario de menor edad del presente estudio coinciden con Barrionuevo \& Solís ${ }^{11}$, donde evidenciaron una RM Clase I con el 62,5\%, seguido de la Clase II, coincidiendo con lo observado por Nallegowda et al..$^{33}$, donde la mayoría de los casos registraron Clase I con el 78\%, asimismo, se observó prognatismo bimaxilar en el $8 \%$ de los casos. Por otro lado, Carmagnani et al. ${ }^{34}$, reportaron un $48 \%$ para la Clase I, seguido de la Clase II con el 33\% y Clase III con el 19\%, del mismo modo, Martínez ${ }^{35}$ registró Clase I (RMD) en el 43,3\%, Clase II en el 40\% (RMD y RMI) y Clase III con menor valor, esto podría deberse al posible cambio en la perspectiva al examinar la hemiarcada izquierda desde la parte derecha del paciente ${ }^{16}$, mientras que los casos con Clase III fueron menores; cabe recalcar que, el presente estudio tuvo como criterio de exclusión a los respiradores bucales, debido a que este síndrome en sí, ocasiona repercusiones en la maloclusión dental y maxilar. Por otro lado, el estudio de Strodel ${ }^{36}$ observó RM Clase II en la mayoría de los casos, expuso que el músculo cuando está espástico puede retrasar el crecimiento de los huesos, además produce variaciones en el tono muscular normal de la cabeza y del cuello, que podrían causar deformidad del arco y compresión del maxilar, similar a lo observado por Sihna et al. ${ }^{29}$, evidenciando mayoritariamente niños PC con RM Clase II en el $58 \%$, asimismo, esta Clase II obtuvo diferencia significativa, mientras que la mordida abierta no lo obtuvo.

De acuerdo al registro de mordida abierta, el presente estudio reportó mayoritariamente $\mathrm{OB}$ negativo en el $32,2 \%$, el grupo de niños no registró $\mathrm{OB}$ en el $33,3 \%$ y el OB fue normal en el $29,4 \%$, se presume que fue NR por el recambio dentario y $\mathrm{OB}$ normal posiblemente a las intervenciones tempranas, mientras que el grupo de adolescente si bien evidenció OB normal en el $40 \%$, observó OB negativo (mordida abierta) en el $24,4 \%$ presumiblemente no tuvieron tratamiento oportuno, Barrionuevo \& Solís ${ }^{11}$, también registraron mayoritariamente mordida abierta en el $63,8 \%$, coincidiendo con el estudio de la Universidad de Washington ${ }^{8}$, donde los niños con PC obtuvieron altos valores, asimismo, Abanto $e t a l .^{31}$, evidenciaron presencia de maloclusiones en el $61.7 \%$, donde incluyen a la mordida abierta y overjet aumentado $\left(>4 \mathrm{~mm}\right.$ ), igualmente Morales ${ }^{37}$ y Sinha et al. ${ }^{29}$, observaron mordida abierta en el $46 \%$ y $25 \%$ de los casos respectivamente. Los resultados obtenidos por Guerreiro \& De Lima ${ }^{26}$, evidenciaron maloclusiones aumentadas en el overbite y overjet en niños con PC, si 
bien el presente estudio observó sólo overjet aumentado en el 36,84\%, no evidenció diferencia significativa entre los grupos etarios, sin embargo, Martínez ${ }^{35}$, observó diferentes maloclusiones: resalte aumentado, mordida abierta, estrechez de arcadas o mordidas cruzadas unilaterales, entre otros. Varios autores, atribuyen que este tipo de maloclusiones se deban a la baja tonicidad de los músculos faciales y movimientos no coordinados de labio y lengua, pero se debe tener en cuenta que, algunos de estos estudios incluyeron respiradores bucales. En el presente estudio, se registró un valor ligeramente alto para línea media dental no coincidente (LMD NC) de $59,8 \%$, similar a lo reportado por Morales ${ }^{37}$, donde evidencia el 73,0\%, destacando que su muestra lo constituyeron niños con PC espástica; asimismo, el presente trabajo mostró LMD desviada hacia la izquierda en el $41,5 \%$ y $19,3 \%$ LMD desviada hacia la derecha, las posibles causas serían: ausencia unilateral de algún diente, discrepancias del tamaño dental, malposición dental y desviación funcional ${ }^{16}$, una hipótesis infiere sobre el potencial genético que induce a un crecimiento dominante del lado derecho o un hipocrecimiento sobre el lado izquierdo de la cara en pacientes que mostraron deformidad facial ${ }^{38}$.

Actualmente, en la revisión de literatura nacional, no se encontraron estudios de salud oral en pacientes con PC con muestras representativas en el grupo etario estudiado ${ }^{28,39,40}$, por lo que, se recomienda realizar estudios de este tipo, en instituciones especializadas. La escasez de trabajos similares posiblemente se deba a la dificultad que presenta el paciente con PC para su evaluación clínica oral, por el tiempo que demande la atención y el gasto económico que pueda originar el número de citas. El presente trabajo formó parte de la tesis doctoral del investigador principal, que sirvió para identificar la realidad de los problemas de salud oral que afronta este tipo de paciente. Su abordaje odontológico, depende del soporte adecuado de los miembros de la familia, ya que la necesidad de asistencia debido a su discapacidad motora aunado al apoyo emocional es mayor, así como el abordaje odontológico en edades tardías dificulta su atención, por lo que, la capacitación del profesional debe ser constante. Se concluye, que el índice de higiene oral simplificado de Greene y Vermillion fue de 2,5, evidenciándose la misma tendencia en ambos grupos etarios que muestra mala higiene oral. La prevalencia de caries fue del 100\%. El índice CPOD fue de 4,55 $\pm 1,557$, observándose el mismo comportamiento en ambos grupos etarios que constituye alto valor de caries. La maloclusión dentaria, predominante para niños fue RM Clase I (RMD 42,1\% y RMI 44,4\%); mientras en adolescentes fue la RM Clase III (RMD 42,2\% y RMI 37,8\%).

\section{Referencias bibliográficas}

1. Redondo N, Scagnet G, Siancha A. Patologías discapacitantes. En: Odontopediatría y Discapacidad. Hospital de Odontología Infantil Don Benito Quinquela Martín -Secretaría de Salud Gobierno de Buenos Aires. Bs Aires. 1999. p. 21-34.
2. Sabbagh-Haddad A., Magalhães MHG. Introdução. In: Sabbagh-Haddad A. et al. Odontologia para Pacientes com Necessidades Especiais. São Paulo: Editora Santos. No prelo 2007; cap. 1, p. 1-5.

3. Vázquez VCC, Vidal RCA. Parálisis cerebral infantil: definición y clasificación a través de la historia. Rev Mex Ortop Ped. [Internet]. 2014 [citado el 16 de octubre de 2020];16(1):6-10. Disponible en: https://www.medigraphic.com/cgi-bin/new/resumen.cgi?IDARTICU$\mathrm{LO}=52957$

4. Quesada L, Fonseca I. La parálisis cerebral como un problema de salud. Correo Científico Médico CMM [Internet]. 2015 [citado el 16 de octubre de 2020]; 19(4):757760. Disponible en: La parálisis cerebral como un problema de salud (sld.cu)

5. Kinsner-Ovaskainen A, Lanzoni M, Delobel-Ayoub M, Ehlinger V, Anaud C, Martin S. Surveillance of Cerebral Palsy in Europe: Development of the JRC-SCPE Central Database and Public Health Indicators. JRC Thechnical Reports. EU Science Hub. [Internet]. 2017 [citado el 16 de octubre de 2020]; DOI: 10.2760/342293. Disponible en: https://publications.jrc.ec.europa.eu/repository/ bitstream/JRC109418/kjna28935enn.pdf

6. Sadowska M, Sarecka-Hujar B, Kopyta I. Cerebral Palsy: Current Opinions on Definition, Epidemiology, Risk Factors, Classification and Treatment Options. Neuropsychiatr Dis Treat [Internet]. 2020 [citado el 16 de octubre de 2020]; 16: 1505-1518. DOI: $10.2147 /$ NDT.S235165. Disponible en: https:/www.ncbi.nlm. nih.gov/pmc/articles/PMC7297454/

7. Oskoui M, Coutinho F, Dykeman J, Jetté N, Pringsheim T. An update on the prevalence of cerebral palsy: a systematic review and meta-analysis. Dev Med Child Neurol [Internet]. 2013 [citado el 16 de octubre de 2020]; 55:509-519. DOI: 10.1111/dmcn.12080. Disponible en: https://pubmed.ncbi.nlm.nih.gov/23346889/

8. University of Washington and Washington State Oral Health Program. Oral Health Fact Sheets for Patients with Special Needs and for Dental Professionals: Children with Cerebral Palsy. Washington, USA, 2010. 5p.

9. Sellier E, McIntyre S, Smithers-Sheedy H, Platt MJ, SCPE and ACPR Groups (2020) European and Australian Cerebral Palsy Surveillance Networks Working Together for Collaborative Research. Neuropediatrics [Internet]. 2020 [citado el 16 de octubre de 2020]; 51(2):105-112. DOI: 10.1055/s-0039-3402003 Disponible en: https://pubmed.ncbi.nlm.nih.gov/31910452/

10. Vila-Nova, F., Oliveira, R., Cordovil, R. (2020). Participation in Leisure Activities by Portuguese Children With Cerebral Palsy. Percept Mot Skills. [Internet]. 2013 [citado el 16 de octubre de 2020]; 127(6):1051-1067. DOI: 10.1177/0031512520931639. Disponible en: https:// pubmed.ncbi.nlm.nih.gov/32552484/

11. Barrionuevo L, Solís FS. Anomalías dentomaxilares y factores asociados en niños con parálisis cerebral. Rev Chil Pediatr [Internet]. 2008 [citado el 16 de octubre de 2020]; 79(3):272-280. Disponible en: http://dx.doi. org/10.4067/S0370-41062008000300005

12. Canut JA. Oclusión normal y maloclusión. En: Ortodoncia Clínica y Terapéutica. 2da ed. Barcelona: Masson; 2005. p. 95-104. 
13. Ramos JR. Ortodoncia y sus diapositivas: Atlas operacional ortholabor. Livraria Santos Editora; 2012. p.13-17.

14. Canut JA. Exploración del paciente. En: Ortodoncia Clínica y Terapéutica. 2da ed. Barcelona: Masson; 2005. p. $105-127$.

15. Ravaglia C. Afecciones que involucran la función cerebral. En: Odontología en niños discapacitados. Metodología para su atención. Bs Aires: Fundación Severo Vaccaro para las Ciencias y el Arte; 1999. p. 81-91.

16. Botero-Mariaca PM, Vélez-Trujillo N. Análisis de Modelos. En: Manual de historia clínica odontológica del escolar. 3ra ed. Bogotá: Universidad Cooperativa de Colombia; 2016. p.195- 225.

17. Vellini-Ferreira F. Oclusión y equilibrio de los dientes. En: Ortodoncia: Diagnóstico y Planificación Clínica. 1 ra ed. Sao Paulo: Ediciones Artes Médicas; 2002. p. 73-96.

18. Falconí EM, Alvarez-Páucar MA, Romero M, Huanqui M, Lay SB, Tanco RL. Prevención en pacientes especiales. En: Melgar RA, Collazos J, Mendoza R, Reyes C. Principios en Prevención de Salud Bucal. Lima, Perú: Fragmento s.a.c.; 2008. p.149-172.

19. De Camargo MA, Antunes JL. Untreated dental caries in children with cerebral palsy in the Brazilian context. Int J Paediatr Dent [Internet]. 2008 [citado el 12 de noviembre de 2020]; 18(2):131-8. DOI: $10.1111 /$ j.1365263X.2007.00829.x. Disponible en: https://pubmed. ncbi.nlm.nih.gov/18237296/

20. Dos Santos MT, Nogueira ML. Infantile reflexes and their effects on dental caries and oral hygiene in cerebral palsy individuals. J Oral Rehabil [Internet]. 2005 [citado el 12 de noviembre de 2020]; 32(12):880-5. DOI: 10.1111/j.1365-2842.2005.01518.x. Disponible en: https://pubmed.ncbi.nlm.nih.gov/16297034/

21. Alvarez-Páucar MA. Influencia de la Alimentación y Deglución en la Maloclusión Dentaria en niños y adolescentes con Parálisis Cerebral que asisten a Centros Especializados, Lima 2012. [Tesis doctoral]. [Lima]: Facultad de Medicina, Universidad Nacional Mayor de San Marcos; 2013.

22. Greene JC, Vermillion JR. The simplified oral hygiene index. J Am Dent Assoc. [Internet]. 1964 [citado el 12 de noviembre de 2020]; 68:7-13. DOI: $10.14219 /$ jada. archive.1964.0034. Disponible en: https://pubmed. ncbi.nlm.nih.gov/14076341/

23. Shulman JD, Cappelli DP. Epidemiology of Dental Caries. In Prevention in Clinical Oral Health Care. Mosby. 2008. p. 2-13 https://doi.org/10.1016/B978-0-32303695-5.50005-7

24. Camacho-Conchucos HT, Fajardo-Campos P, Zavaleta 1 . Análisis descriptivo sobre deficiencias y discapacidades del desarrollo psicomotor en pacientes atendidos en el Instituto Nacional de Rehabilitación 2006 - 2008. An. Fac. Med. [Internet]. 2012 [citado 2020 Nov 30] ; 73(2):119-126. Disponible en: http:// www.scielo.org.pe/scielo.php?script=sci_arttext\&pi$\mathrm{d}=$ S1025-55832012000200007\&lng=es.

25. Castellanos JL, Díaz LM, Gay O. Enfermedades Neuropsiquiátricas. En: Lazo M Editor. Medicina en Odontología: manejo dental de pacientes con enfermedades sistémicas. 2a ed. México: Manual Moderno, 2002 p. 235-259.

26. Guerreiro OP, De Lima GG. Diagnóstico das condiçóes de saúde bucal em portadores de paralisia cerebral do município de Pelotas, Rio Grande do Sul, Brasil. Cien. saúde coletiva [Internet]. 2009 [citado el 16 de octubre de 2020]; 14(5):1-10. Disponible en: https://doi. org/10.1590/S1413-81232009000500036

27. III Curso Internacional - II Jornada Nacional ASPODES 2010: Retos en tratamiento dental, con énfasis entranstornos neuropsicomotores. Centro Cultural Ricardo Palma Miraflores. 7-9 octubre 2010. Lima: Asociación peruana de Odontoestomatología de Pacientes Especiales; 2010.

28. Soto RA, Vallejos RE, Falconi EM, Monzón F. Patologías bucales en niños con encefalopatía infantil en el Perú. Rev Estomatol Herediana [Internet]. 2006 [citado el 16 de octubre de 2020]; 16(2):115-119. Disponible en: https://revistas.upch.edu.pe/index.php/REH/article/ view/1915/1922

29. Sinha N, Singh B, Chhabra KG, Patil S. Comparison of oral health status between children with cerebral palsy and normal children in India: A case-control study. J Indian Soc Periodontol [Internet]. 2015 [citado el 16 de octubre de 2020]; 19(1):78-82. DOI: 10.4103/0972124X.145800. Disponible en: https:/www.ncbi.nlm. nih.gov/pmc/articles/PMC4365163/

30. Cardoso AM., Gomez LN, Silva CR, Soares R, Abreu MH, Padilha WW et al. Dental Caries and Periodontal Disease in Brazilian Children and Adolescents with Cerebral Palsy. Int J. Environ. Res. Public Health [Internet]. 2015 [citado el 16 de noviembre de 2020]; 12: 335-353. DOI: 10.3390/ijerph120100335. Disponible en: https://www.ncbi.nlm.nih.gov/pmc/articles/ PMC4306865/

31. Abanto J, Oliveira A, Prócida D, Bönecker M, Medeiros F, Ciamponi AL. Impact of oral diseases and disorders on oral-health-related quality of life of children with cerebral palsy. Spec Care Dentist [Internet]. 2014 [citado el 16 de octubre de 2020]; 34(2):56-63. DOI: 10.1111/ scd.12028. Disponible en: https://pubmed.ncbi.nlm. nih.gov/24588489/

32. Alvarez MA. Parálisis cerebral y maloclusión dentaria en niños y adolescentes. Rev Peru Pediatr. 2013;66(4):234241.

33. Nallegowda M, Mathur V, Singh U, Prakash H, Khanna M, Sachdev G, et al. Oral health status in Indian children with cerebral palsy - A pilot study. IJPMR [Internet]. 2005 [citado el 16 de octubre de 2020]; 16(1):14. Disponible en: https://citeseerx.ist.psu.edu/viewdoc/ download?doi=10.1.1.602.6665\&rep=rep1 \&type=pdf

34. Carmagnani GF, De Mattos GGK, Correa PMS, Rodrigues DMT. Occlusal Characteristics in Cerebral Palsy Patients. Journal of Dentistry for Children IJPMR [Internet]. 2007 [citado el 16 de octubre de 2020]; 74(1):41-45. Disponible en: https://citeseerx.ist.psu. edu/viewdoc/download?doi=10.1.1.602.6665\&rep=rep1\&type $=$ pdf

35. Martínez VI. Análisis de la Maloclusión y su Relación con la Posición de Reposo de la Cabeza y las Funciones Orales en un Grupo de Pacientes con Parálisis Cerebral 
Comparado con un Grupo Control. 2015 [Tesis doctoral]. [Valencia]: Facultat de Medicina i Odontología, Universidad de Valencia; 2015. 194 p.

36. Strodel DJ. The effects of spastic cerebral palsy on occlusion. J Dent Child [Internet]. 1987 [citado el 16 de octubre de 2020]; 54 (4): 255-260. Disponible en: https:// pubmed.ncbi.nlm.nih.gov/3476527/

37. Morales AP. Frecuencia de maloclusiones en niños con parálisis cerebral infantil leve de tipo espástica 7-14 años de edad del Hogar Clínica San Juan de Dios. [Tesis de bachiller]. [Lima]: Facultad de Odontología, Universidad Nacional Mayor de San Marcos; 2002.

38. Servet T, Proffit W. The prevalence of facial asymmetry in the dentofacial deformities population at the University of North Carolina. Int J Adult Orthodon Orthognath Surg, 1997; 12: 171-176.
39. Vila F. Higiene Bucal y Caries Dental en niños menores de 5 ańos con Parálisis Cerebral en el Instituto Nacional de Salud del Nińo - 2017. [Tesis de pregrado] [Lima]: Facultad de Odontología, Universidad Nacional Federico Villarreal, 2018

40. Segura CA. Anomalías Dentomaxilares asociado a Parálisis Cerebral en niños de 2 a 12 años de edad en el Instituto para el Desarrollo Infantil - ARIE. [Tesis de pregrado]. [Lima]: Facultad de Odontología: Universidad de San Martin de Porres, 2012. 